Ger J Exerc Sport Res 2022 · 52:148-158 https://doi.org/10.1007/s12662-021-00769-9 Eingegangen: 31. März 2021

Angenommen: 23. September 2021

Online publiziert: 18 . Oktober 2021

(c) Der/die Autor(en) 2021

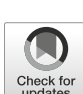

Niklas Kramer (D) - Claas Wegner (D)

Biologiedidaktik, Universität Bielefeld, Bielefeld, Deutschland

\title{
Gemäßigt konstruktivistische Prozessmerkmale fächerübergreifenden Unterrichts im Fächerverbund Sport und Biologie
}

Sportunterricht von einer potenziellen Steigerung kognitiver Aktivierung der Schüler*innen (Balz, 2021). Trotz dieser vielversprechenden Argumente stellt sich die Erforschung fächerübergreifenden Unterrichts an der Schnittstelle zum Sportunterricht sehr eindimensional und limitiert dar, da in aktuellen Studien vorrangig der Wissenserwerb untersucht wird und weitere theoretisch diskutierte Auswirkungen fächerübergreifenden Unterrichts wie u.a. affektive Faktoren kaum erforscht werden (Kramer \& Wegner, 2020a). Demnach muss ein fächerübergreifend orientierter Sportunterricht primär in seinen strukturellen Merkmalen und hinsichtlich seiner potenziellen Auswirkungen auf die Schüler*innen und Lehrer*innen stärker empirisch im Vergleich zum Fachunterricht untersucht werden, um den didaktischen Diskurs über eine vermehrte Umsetzung nicht allein auf der Grundlage konzeptioneller Ideen, sondern zusätzlich durch empirische Befunde zu beleben (Balz, 2021; Kramer \& Wegner, 2020a). In diesem Artikel soll vor allem ein strukturell orientierter Blick auf einen fächerübergreifenden Sportunterricht geworfen werden. Nach Labudde (2003) unterscheidet sich ein Fachgrenzen überschreitender Unterricht $u$. a. in seinen konstruktivistischen Prozessmerkmalen vom Fachunterricht. Knüpfen Schüler*innen an ihr Vorwissen an, das nicht in Fachdisziplinen getrennt vorliegt, und konstruieren aktiv neues Wissen in subjektiv rele- vanten Kontexten, kann dies nicht in einer Fachdisziplin geschehen und muss notwendigerweise Fachgrenzen überwinden, um relevante Fragestellungen beantworten zu können. Ein solcher Unterricht sei demnach situativer, aktiver und konstruktiver (Labudde, 2003). Inwiefern sich ein fächerübergreifender Unterricht in diesen Merkmalen vom Fachunterricht abgrenzt, wird in der vorliegenden Studie näher untersucht. Dies dient einer ersten Charakterisierung eines fächerübergreifend orientierten Sportunterrichts und bietet zudem Anknüpfungspunkte an potenziell positive Auswirkungen für den Sportunterricht und damit zusammenhängend auch für die Schüler*innenschaft.

\section{Kognitive Aktivierung im Sportunterricht}

Die Erforschung eines mehrperspektivischen Sportunterrichts erhält im aktuellen Diskurs um die kognitive Aktivierung im Sportunterricht einen immer größeren Stellenwert und eine empirische Legitimation. Unter kognitiver Aktivierung wird eine von drei Basisdimensionen (kognitive Aktivierung, effektive Klassenführung, unterstützendes Unterrichtsklima) verstanden, die durch Ergebnisse der empirischen Bildungsforschung als besonders bedeutsam für die Unterrichtsqualität im Sinne eines motivierenden und lernwirksamen Unterrichts herausgestellt werden (Lipowsky \& Hess, 2019). Nach 
Lipowsky (2015) ist unter kognitiver Aktivierung, die Anregung der Lernenden „zum vertieften Nachdenken und $\mathrm{zu}$ einer elaborierten Auseinandersetzung mit dem Unterrichtsgegenstand “ zu verstehen (S. 89). Anders ausgedrückt ist hiermit die Intention gemeint, durch komplexe Aufgaben kognitive Aktivität, "also subjektive Konstruktionsprozesse“ (Sygusch, Hapke, Liebl, \& Töpfer, 2021, S. 170), bei den Schüler*innen auf einem lernwirksamen Niveau durch die Lehrkraft anzuregen (Hermann, Seiler, \& Niederkofler, 2016; Niederkofler \& Amesberger, 2016; Sygusch et al., 2021). Sie ist daher für das fachliche Wissen sowie das Verstehen und die Beurteilung von Sachverhalten relevant und stellt das Fach Sport vor eine Herausforderung, da es neben einer kognitiven, vielmehr durch eine physische Aktivität gekennzeichnet ist (Balz, 2021). Es stellt sich somit die Frage nach der generellen Umsetzbarkeit kognitiver Aktivierung im Sportunterricht, da häufig nur bestimmte Fertigkeiten erlernt werden, die kognitiv nicht interpretiert werden müssen (Balz, 2021; Messmer, 2021). Allerdings ist es im Sportunterricht ebenfalls wichtig, die sportliche Tätigkeit zu verstehen (Messmer, 2021). Eine mögliche Perspektive lässt sich daher über einen mehrperspektivischen Sportunterricht einnehmen, der eine Betrachtung des Sports aus verschiedenen Perspektiven zulässt und komplexe Phänomene erschließen kann (Balz, 2021). Einem solchen Unterricht ist die kognitive Aktivierung inhärent, da die Einnahme von und das Wechseln zwischen unterschiedlichen Perspektiven Kognitionen aufbauen und verändern (Balz, 2021). Kontrastierend besteht eine unzureichende Realisierung von Unterrichtskonzepten zur kognitiven Aktivierung und ein Evaluationsdefizit eines speziell mehrperspektivischen Sportunterrichts, dem mit der vorliegenden Studie begegnet werden soll (Balz, 2021).

Den theoretischen Hintergrund der kognitiven Aktivierung bilden konstruktivistische Lerntheorien, die eine eigenständige Konstruktion neuen Wissens betonen (Lipowsky, 2015; Lipowsky \& Hess, 2019; Sygusch et al., 2021).
Die vorliegende Studie untersucht daher einen fächerübergreifenden Unterricht im Fächerverbund Sport und Biologie im Vergleich zu den Einzelfächern bezüglich ihrer konstruktivistischen Prozessmerkmale. Darauf aufbauend sollten sich weitere Studien zur Eruierung der Effektivität (bspw. affektiv-motivationale Aspekte, kognitive Aktivierung) anschließen.

\section{Gemäßigter Konstruktivismus}

Wie bereits erörtert, entspringt die kognitive Aktivierung einer konstruktivistischen Lerntheorie, die als Grundlage der Studie fungiert.

Zunächst sei sie von der erkenntnistheoretisch geprägten Form des radikalen Konstruktivismus von Glaserfeld (1984) abgegrenzt. Diese Theorie beschreibt die Aneignung von Wissen immer als einen aktiven und individuellen Konstruktionsprozess. Die Wirklichkeit kann durch die eigenen Sinnesleistungen und durch das Vorwissen eines jeden Individuums nicht ganzheitlich und objektiv erfasst werden, weswegen auch kein geteiltes Wissen zwischen Individuen möglich ist (Glaserfeld, 1984). Diese Position erschwert den Austausch im didaktischen Segment. Dort bietet der lerntheoretische Ansatz von Reinmann und Mandl (2006) gute Anknüpfungspunkte, da sie sich für eine gemäßigte Position aussprechen, in der geteiltes Wissen sowie Instruktionen möglich sind. Instruktionen gänzlich durch offene Lernumgebungen im Sinne einer individuellen und aktiven Wissenskonstruktion jedes Lernenden zu substituieren, führt zu einer Überforderung zumeist schwächerer Schüler*innen und $\mathrm{zu}$ einer geringen Lenkung des Unterrichts (Reinmann \& Mandl, 2006). Gerade unter Betrachtung dieser Gefahr muss das Vorwissen der Schüler*innen sehr genau beachtet und für die Gestaltung der Lernumgebung miteinbezogen werden. Reinmann und Mandl (2006) sprechen sich in der Umsetzung für eine Problemorientierung als zentrales Leitprinzip ihres gemäBigten Konstruktivismus aus. Für die Aneignung des benötigten Fundaments postulieren Reinmann und Mandl (2006) eine Unabdingbarkeit der Instruktion.
Doch wie gestaltet sich ein konstruktivistischer Lernprozess im Sinne des gemäßigten Konstruktivismus? Reinmann und Mandl (2006) stellen hierzu sechs Prozessmerkmale des Lernens auf:

Innerhalb eines effektiven Lernprozesses sollte der Lernende eine aktive Rolle einnehmen, da Lernen selbst auch immer ein aktiver Prozess ist (Terhart, 1999; Reich, 2005). Dieser Prozess kann von außen lediglich angestoßen, aber nie gesteuert werden (Terhart, 1999).

Im Sinne eines selbstgesteuerten Lernens müssen die Lernenden ihre Lernprozesse selbstverantwortlich steuern und kontrollieren können (Desch, Basten, Großmann, \& Wilde, 2017). Dies ist je nach Lernsituation in verschiedener Stärke möglich (Reinmann \& Mandl, 2006).

Das Lernen stellt immer einen konstruierenden Prozess dar (Reinmann \& Mandl, 2006). Das Vorwissen sowie die Fertigkeiten und Fähigkeiten der Lernenden bilden dabei die Grundlage, in die der neue Inhalt integriert und so neues Wissen konstruiert wird (Desch et al., 2017).

Ein Lernprozess ist immer emotional geprägt (Reinmann \& Mandl, 2006). Positive Emotionen (Freude, Interesse) können dem Lernprozess dienlich sein, während negativ konnotierte Emotionen (Frustration, Angst) eine hemmende Wirkung haben können (Urhahne, Marsch, Wilde, \& Krüger, 2011).

Lernprozesse sind zudem immer $s i$ tuativ an bestimmte Kontexte gebunden (Reinmann \& Mandl, 2006). Es ist zu empfehlen, möglichst authentische und alltagsnahe Kontexte der Lernenden zu wählen (Desch et al., 2017).

Zuletzt ist ein Lernprozess immer durch eine soziale Komponente geprägt, da sich Lernen meist in Interaktion mit anderen Lernenden vollzieht (Reinmann \& Mandl, 2006).

Diese lerntheoretische Position des gemäßigten Konstruktivismus sei leitender Gedanke der vorliegenden Studie, in der die Prozessmerkmale spezifischer untersucht werden. 
Ger J Exerc Sport Res 2022 · 52:148-158 https://doi.org/10.1007/s12662-021-00769-9

(c) Der/die Autor(en) 2021

\section{N. Kramer · C. Wegner}

\section{Gemäßigt konstruktivistische Prozessmerkmale fächerübergreifenden Unterrichts im Fächerverbund Sport und Biologie}

\section{Zusammenfassung}

Sportunterricht wird in vielen konzeptionell-reflektorischen Arbeiten als ein ergiebiger Partner für fächerübergreifende Unterrichtsmaßnahmen gesehen. Ein solch mehrperspektivisch orientierter Sportunterricht bietet im Hinblick auf den sportpädagogischen Diskurs eine Möglichkeit, kognitive Aktivität im Schulsport zu implementieren und die Relevanz des Faches zu erhöhen. Dennoch zeigt sich ein Evaluationsdefizit eines solchen Unterrichts. Die vorliegende Studie wirkt diesem Defizit entgegen und setzt sich zunächst mit strukturellen Merkmalen eines fächerübergreifenden Unterrichts auseinander. Da sowohl das Konstrukt der kognitiven Aktivierung als auch der fächerübergreifende Unterricht eine Nähe zum Konstruktivismus offenbaren, soll in der vorliegenden Studie untersucht werden, inwiefern sich ein fächerübergreifender
Unterricht in den konstruktivistischen Prozessmerkmalen (situativ, aktiv, konstruktiv) vom Fachunterricht unterscheidet. Dafür nahmen insgesamt $N=75$ Schüler*innen (15,2 Jahre) aus vier Kursen des Sekundarbereichs an einer fächerübergreifenden Unterrichtseinheit im Fächerverbund Sport und Biologie teil. Zur Evaluation der konstruktivistischen Prozessmerkmale wurde der Kurzfragebogen nach Basten et al. eingesetzt, der für den fächerübergreifenden Unterricht sowie für den Sport- und Biologieunterricht bewertet wurde. Messwiederholte Varianzanalysen zeigten signifikante Unterschiede zwischen den fächerübergreifenden Unterrichtseinheiten und dem Biologieunterricht in allen drei Subskalen. Der Sportunterricht unterschied sich lediglich in der Subskala "situativ" von den Unterrichtseinheiten. Der Einfluss des subjektiven Vorwissens auf die Bewertung der fächerübergreifenden Unterrichtseinheiten konnte nur für die Subskala "konstruktiv" festgestellt werden. Schüler*innen mit viel Vorwissen bewerteten diese im fächerübergreifenden Zusammenhang signifikant höher. Die Studie stützt damit die bereits theoretisch angenommene höhere Ausprägung der prozessbezogenen Merkmale des gemäßigten Konstruktivismus in fächerübergreifenden Unterrichtseinheiten im Vergleich zum Biologieunterricht. Zudem wird die Sonderstellung des Faches Sport deutlich, die in weiteren Studien untersucht werden sollte.

\section{Schlüsselwörter}

Interdisziplinär · Interventionen · Oberstufe · Kognitive Aktivierung · Vorwissen

\section{Moderate constructivist process characteristics of interdisciplinary interventions in the combined subject of physical education and biology}

\begin{abstract}
In many conceptual-reflective papers, the use of physical education is seen as advantageous when designing interdisciplinary interventions. Interdisciplinary physical education offers several possibilities to implement cognitive activity into physical education and increase the subject's relevance. Nevertheless, evaluations regarding these teaching approaches are lacking. This study addresses this deficit by exploring structural characteristics of interdisciplinary teaching. Since both cognitive activity and interdisciplinary teaching are connected to constructivism, the present study investigates the extent to which interdisciplinary teaching differs in constructivist process characteristics
\end{abstract}

(situational, active, constructive) from single subjects. A total of 75 students aged 15.2 years from four different secondary school courses participated in an interdisciplinary intervention combining physical education and biology. A questionnaire based on a study by Basten et al. was used to evaluate constructivist process characteristics, which was assessed for the intervention as well as the physical education and biology courses. A repeated measures analysis of variance (ANOVA) concluded that in comparison to regular biology courses, students reported significantly higher values for all three subscales, whereas physical education only differed in the subscale "situational". In addition, we examined the influence of subjective prior knowledge, where only the "constructive" subscale was rated significantly higher in students with more prior knowledge. Our study suggests that interdisciplinary interventions exhibit a higher degree of process characteristics in moderate constructivism compared to biology classes. In addition, a special position of physical education is supported by the results, which should be examined in further studies.

Keywords Interdisciplinary · Interventions · Secondary school - Cognitive activation . Prior knowledge

\section{Konstruktivismus im Sport- und Biologieunterricht}

Im Sportunterricht geht es um die Lösung motorischer Probleme, die nicht selten aus dem Alltag der Schüler*innen (Balancieren auf einer Wegbegrenzung o. ä.) stammen (Brodtmann \& Landau, 1982). Diese Probleme sind situativ nahe an den Alltagserfahrungen der
Schüler*innen und bieten damit eine günstige Ausgangslage für einen konstruktivistisch orientierten Unterricht. Bei der Lösung der Probleme nimmt der Sportunterricht eine Sonderstellung im Vergleich zu den meisten Fächern ein, denn Probleme können hier anders als in kognitiv orientierten Fachdisziplinen nicht von anderen Schüler*innen für die eigene Person gelöst werden (Brodt- mann \& Landau, 1982). Neben den Bewegungsproblemen seien ebenfalls jene zu nennen, die eine fächerübergreifende Auseinandersetzung verlangen (Brodtmann \& Landau, 1982). Daher gibt es Bestrebungen zu einer stärker kognitiv aktivierenden Herangehensweise (s. Kognitive Aktivierung im Sportunterricht). Denn Sportunterricht ist wichtig, um Schüler*innen neben der Bewälti- 


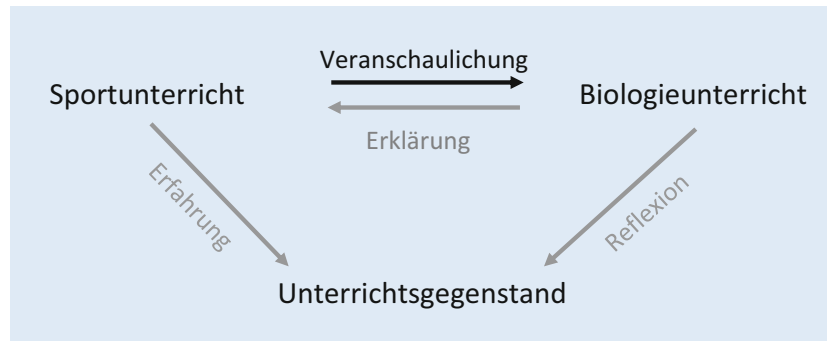

Abb. $1<$ Beziehungsdreieck der Fächer Biologie und Sport zum Unterrichtsgegenstand. (Mod. nach Ukley et al., 2013) gung von Bewegungsproblemen auch über die Wichtigkeit eines gesunden und aktiven Lebens aufzuklären (Sun, Chen, Zhu, \& Ennis, 2012). Davon ausgehend, entwickelten sich vorrangig im amerikanischen Raum sportpädagogische Ansätze, die sich auf einen sozialen Konstruktivismus stützen und Wissen für ein langes und gesundes Leben vermitteln (Azzarito \& Ennis, 2003). Die zusätzliche kognitive Komponente erweist sich als ein Bindeglied zwischen der physischen Erfahrung und der Bedeutung für das eigene Leben (FernándezBalboa, 1997). Der Sportunterricht erhält hierdurch einen höheren Stellenwert und gewinnt an Relevanz. Bisherige Studienergebnisse legen positive Effekte dieses konstruktivistischen Ansatzes nahe. Demnach steigert er die Motivation für den Sportunterricht aber auch für die Ausübung außerschulischen Sports und hat einen positiven Einfluss auf den Erwerb gesundheitsrelevanten Wissens (Chen, Martin, Sun, \& Ennis, 2007; Chen, Sun, Zhu, \& Chen, 2014; Zhang et al., 2014). Die Kombination von physischen und kognitiven Komponenten weist starke Parallelen zum fächerübergreifenden Unterricht und zum Ansatz der kognitiven Aktivierung auf, dem durch die empirische Bildungsforschung ebenfalls ein hoher Stellenwert zukommt. Die positiven Befunde forcieren eine weitere empirische Auseinandersetzung mit dem Konzept eines fächerübergreifenden Sportunterrichts.

Das Fach Sport bietet hierfür eine einzigartige Voraussetzung - den Erfahrungswert (Ukley et al., 2013). Wie in keinem anderen Fach können Schüler*innen durch körperliche Aktivität Primärerfahrungen machen, wodurch das theoretische Wissen auf einer neuen Ebene erlebbar wird (Ukley et al., 2013). Die Biologie bietet eine gute Ergänzung zum Sport, da sie besonders vor dem Hintergrund der Gesundheitserziehung und der Anpassungsmechanismen eines Trainings die notwendigen theoretischen Hintergründe liefert. Das Charakteristikum der Problemorientierung moderat konstruktivistischer Unterrichtsvorhaben ist immanenter Bestandteil des Faches Biologie (Ministerium für Schule und Weiterbildung des Landes Nordrhein-Westfalen, 2014, 2019). Der naturwissenschaftliche Erkenntnisweg basiert auf einer problemorientierten Ausgangslage. Dennoch lässt sich nicht pauschalisieren, ob die sechs Merkmale der konstruktivistischen Lerntheorie nach Reinmann und Mandl (2006) im Unterricht stark ausgeprägt sind, da das Problem nicht zwangsweise auf den Erfahrungen der Schüler*innen beruht (situativ) und nicht unbedingt ein Konstruktionsprozess angestoßen wird. Es gibt aber empirische Hinweise, dass ein Biologieunterricht von Schüler*innen als konstruktivistisch wahrgenommen werden kann (Marsch, Hartwig, \& Krüger, 2009).

Zusammenfassend kann ein Biologieunterricht durch den naturwissenschaftlichen Erkenntnisweg und ein Sportunterricht durch Bewegungsprobleme bereits in seinen Fachgrenzen durch konstruktivistische Prozessmerkmale gekennzeichnet sein. In Form fächerübergreifender Unterrichtsvorhaben lassen sich die beiden Fachdisziplinen darüber hinaus idealtypisch miteinander verbinden. Durch den erörterten Erfahrungswert des Sportunterrichts sammeln die Schüler*innen Primärerfahrungen, die durch theoretische Inhalte der Biologie erklärt werden können. Gleichzeitig wird dieses Wissen durch den Sportunterricht veranschaulicht und erlebbar gemacht, wodurch nicht nur das Fach Sport, sondern auch die Biologie ent- scheidend von einer Fächerverknüpfung profitiert (• Abb. 1). Es wird nicht nur träges Wissen vermittelt, sondern eine aktive Auseinandersetzung mit dem Gegenstand ermöglicht.

\section{Konstruktivistische Prozess- merkmale fächerübergreifen- den Unterrichts}

Im fächerübergreifenden Unterricht werden Problemstellungen über den Zugang durch mehrere Fächer bearbeitet. An die Ideen des gemäßigten Konstruktivismus anlehnend, können Schüler ${ }^{\star}$ innen bestehendes Wissen mit neuen Inhalten verknüpfen und dieses unter Berücksichtigung verschiedener disziplinärer Zugänge und Perspektiven erweitern. Im Fachunterricht ist das in dieser Tragweite nicht möglich. Dies lässt sich wie folgt begründen:

Ein konstruktivistisch geprägter Unterricht, der sich als primär aktiv, situativ und konstruktiv versteht, argumentiert Labudde (2003), muss zwangsläufig fächerübergreifend orientiert sein. Werden Lernende dazu aufgefordert, , an ihr Vorverständnis anzuknüpfen und neues Wissen aktiv in einem für sie relevanten Kontext aufzubauen" (Labudde, 2003, S. 50), kann dies nicht in den Fachgrenzen stattfinden, sondern muss fachüberschreitende Phasen beinhalten. In dieser Argumentation wird bereits deutlich, dass auch im fächerübergreifenden Unterricht die lernrelevanten Kontexte bei den Lernenden gesucht werden, um sie in ihrer Lebenswirklichkeit und in ihren verschiedenen Lernvoraussetzungen abzuholen (Labudde, 2008). Diese Kontexte sollen ebenfalls möglichst authentische Probleme darstellen, damit die Schüler*innen lernen, Problemsituationen aus verschiedenen Perspektiven zu betrachten. Dennoch wird zur Lösung dieser Probleme ein Grundwissen benötigt, welches in der notwendigen Tiefe vorrangig im Fachunterricht erarbeitet werden kann (Popp, 1997). Demnach resultiert die Debatte um den fächerübergreifenden Unterricht in der Empfehlung, ein Gleichgewicht zwischen übergreifenden und fachspezifischen Phasen im Unterricht zu wahren (Moegling, 2010). 


\section{Hypothesen}

Die angeführten Prozessmerkmale des gemäßigten Konstruktivismus eines fächerübergreifenden Unterrichts sind theoretisch begründet. In der vorliegenden Studie soll untersucht werden, ob sich die Prozessmerkmale eines gemäßigten konstruktivistischen Unterrichts im fächerübergreifenden Unterricht SportBiologie auf Basis der Einschätzungen von Schüler*innen empirisch belegen lassen. Die in den vorherigen Kapiteln angeführte Argumentation spricht für eine stärkere Ausprägung konstruktivistischer Prozessmerkmale innerhalb fächerübergreifender Maßnahmen. Labudde (2003) stellt in seiner Argumentation vor allem die konstruktivistischen Merkmale „aktiv“, „situativ“ und „konstruktiv“ als Stärken fächerübergreifenden Unterrichts dar. Aus diesem Grund werden die drei Merkmale genauer untersucht und mit dem Fachunterricht verglichen. Dabei ist der Vergleich zum Sportunterricht besonders interessant, da dieser hinsichtlich der angesprochenen Merkmale eine Nähe zum fächerübergreifenden Unterricht aufweist. Folgende Annahmen wurden gebildet:

- H1: Das Prozessmerkmal „aktiv“ wird von den Lernenden in den fächerübergreifenden Unterrichtseinheiten höher bewertet als in den beiden Einzelfächern Biologie und Sport.

- H2: Das Prozessmerkmal „situativ“ wird von den Lernenden in den fächerübergreifenden Unterrichtseinheiten höher bewertet als in den beiden Einzelfächern Biologie und Sport.

- H3: Das Prozessmerkmal „konstruktiv“" wird von den Lernenden in den fächerübergreifenden Unterrichtseinheiten höher bewertet als in den beiden Einzelfächern Biologie und Sport.

Innerhalb des didaktischen Diskurses über konstruktivistischen Unterricht wird dem Vorwissen eine große Bedeutung für das Gelingen eben jener Unterrichtsmaßnahmen zugesprochen. Es wird angenommen, dass das Vorwissen der Lernenden einen Einflussfaktor auf die Wahrnehmung der konstruktivistischen Merkmale darstellt. Demnach wird folgende Hypothese aufgestellt:

- H4: Lernende mit einem subjektiv hohen Vorwissen bewerten die konstruktivistischen Prozessmerkmale der fächerübergreifenden Unterrichtseinheiten höher als Schüler*innen mit einem subjektiv geringen Vorwissen.

\section{Methode}

\section{Stichprobe}

An der Studie nahmen 75 Schüler*innen aus vier Kursen des Sekundarbereichs teil. Das durchschnittliche Alter der Kursteilnehmer betrug 15,2 Jahre. Die Teilnahme an der Studie war freiwillig. Alle Kurse nahmen an einer fächerübergreifenden Unterrichtseinheit teil, in der die Fächer Biologie und Sport miteinander verbunden wurden. Es gab zwei thematisch verschiedene Konzepte, die wiederum entweder als Tagesmaßnahme oder als Unterrichtsreihe durchgeführt wurden. Biologisch waren beide im Bereich Neurobiologie zu verorten, und sportwissenschaftlich beinhalteten die Maßnahmen Wissen über Koordination und Trainingslehre. Trotz der Differenzen folgten alle Maßnahmen demselben methodischen Gang, der im Sinne fächerübergreifenden Unterrichts die Prozessmerkmale des gemäßigten Konstruktivismus (aktiv, situativ, konstruktiv) nach Reinmann und Mandl (2006) aufwies.

\section{Fächerübergreifender Unterricht}

Die Darstellung des Unterrichtsaufbaus erfolgt zunächst allgemein und dann an einem konkreten Beispiel mit Bezügen zu den Prozessmerkmalen des gemäßigten Konstruktivismus nach Reimann und Mandl (2006).

$\mathrm{Zu}$ Beginn jeder Unterrichtseinheit wurde ein sportbiologisches Problem durch eine kleine sportpraktische Einheit entwickelt. Diese Probleme waren so ausgewählt, dass sie möglichst authentisch waren und einen Alltagsbezug herstellten (situativ). Zur jeweiligen Fragestellung sollten die Schüler*innen Hypothesen entwickeln, die anschlie- ßend aus biologischer und sportpraktischer Sichtweise überprüft wurden. Die Erarbeitungen der grundlegenden theoretischen Konzepte wurden methodisch in Gruppenarbeiten organisiert. Mit Hilfe von u. a. Modellen, psychologischen Testverfahren oder Messgeräten konnten sich die Schüler*innen die Inhalte selbst aneignen und sie am eigenen Körper erleben (aktiv). Dabei half ihnen ihr Vorwissen, auf dem sie immer weiter aufbauen konnten (konstruktiv). In Zwischensicherungen und einer abschließenden Diskussion konnten die jeweiligen Erkenntnisse im Hinblick auf das Eingangsproblem überprüft werden (situativ). Das theoretisch erarbeitete Wissen wurde daraufhin zur Beurteilung eines sportpraktischen Trainings herangezogen (aktiv, konstruktiv). Abschließend erfolgte der Bezug zur Theorie und zur anfänglichen Problemstellung, um Konsequenzen für den Alltag der Schüler*innen abzuleiten (situativ).

In der Unterrichtseinheit zum Thema „Lernen durch Bewegung“ wird zu Beginn eine koordinative Übung vorgestellt (Tennisbälle parallel hochwerfen, Arme kreuzen, Bälle fangen). Trotz der leichten Teilübungen (Werfen, Kreuzen, Fangen) stellt die kombinierte Durchführung eine größere Schwierigkeit dar. Zur vollständigen Klärung der Frage „Warum fällt uns diese koordinative Übung so schwer?" bedarf es fachüberschreitender Momente. Erste Ideen zielen häufig auf Probleme des Gehirns oder der Reizweiterleitung. Daher erfolgt eine Erarbeitung verschiedener Gehirnareale und ihrer Funktionen bei der Durchführung von Bewegungen. Dabei greifen die Schüler*innen auf Wissen zum Aufbau des Gehirns und zur Reizweiterleitung zurück (konstruktiv). Abschließend werden das Assoziationsareal Frontallappen und die dort lokalisierten exekutiven Funktionen herausgestellt, welche die Ausführung koordinativ anspruchsvoller Übungen erschweren. Die Lernenden erleben die Auswirkungen der exekutiven Funktionen zunächst über kleine psychologische Testverfahren (u.a. Stroop, Eriksen Flanker) und dann in einem kombinierten Training aus kognitiven und sportpraktischen Elementen (aktiv). Bevor die Schüler ${ }^{\star}$ innen ein kombiniertes 
Tab. 1 Interne Konsistenzen, Beispiel-Item und Item-Anzahl der jeweiligen Prozessmerkmale des gemäßigten Konstruktivismus (aktiv, situativ, konstruktiv) in Abhängigkeit der jeweiligen Unterrichtssettings ( $N=75)$. (Mod. nach Basten et al. (2015))

\begin{tabular}{|c|c|c|c|c|}
\hline Subskala & Beispiel-Item & $\begin{array}{l}\text { Item- } \\
\text { Anzahl }\end{array}$ & Unterrichtssetting & $\begin{array}{l}\text { Cronbachs } \\
\text { Alpha }\end{array}$ \\
\hline \multirow[t]{3}{*}{ Aktiv } & \multirow{3}{*}{$\begin{array}{l}\text {... war ich beim Ler- } \\
\text { nen tätig }\end{array}$} & \multirow[t]{3}{*}{3} & Fächerübergreifender Unterricht & 0,852 \\
\hline & & & Biologieunterricht & 0,903 \\
\hline & & & Sportunterricht & 0,839 \\
\hline \multirow[t]{3}{*}{ Situativ } & \multirow{3}{*}{$\begin{array}{l}\ldots \text { habe ich etwas } \\
\text { gelernt, was mir im } \\
\text { Leben weiterhilft }\end{array}$} & \multirow[t]{3}{*}{3} & Fächerübergreifender Unterricht & 0,808 \\
\hline & & & Biologieunterricht & 0,903 \\
\hline & & & Sportunterricht & 0,875 \\
\hline \multirow[t]{3}{*}{ Konstruktiv } & \multirow{3}{*}{$\begin{array}{l}\ldots \text { habe ich auf vor- } \\
\text { handenes Wissen } \\
\text { aufgebaut }\end{array}$} & \multirow[t]{3}{*}{4} & Fächerübergreifender Unterricht & 0,908 \\
\hline & & & Biologieunterricht & 0,936 \\
\hline & & & Sportunterricht & 0,873 \\
\hline
\end{tabular}

Training durchführen, werden seine biologischen Auswirkungen auf das Gehirn (neuronale Plastizität, Langzeitpotenzierung) erörtert. Es wird deutlich, dass sich durch ein solches Training nicht allein die Koordination verbessert, sondern generell kognitive Prozesse besser ablaufen können (situativ). In dem Praxisteil werden verschiedene Spiele durchgeführt, die jeweils eine exekutive Funktion ansprechen. Mit Rückgriff auf das erworbene Wissen benennen die Lernenden die jeweiligen Funktionen und verändern selbstständig Spiele, sodass andere exekutive Funktionen gefordert und gefördert werden (aktiv, konstruktiv).

Eine ausführlicher beschriebene Unterrichtsreihe kann bei Kramer et al. (im Druck) nachvollzogen werden.

\section{Testinstrument}

Das verwendete Testinstrument geht auf die Arbeiten von Urhahne et al. (2011) und Basten et al. (2015) zurück. Die Wissenschaftler*innen nutzen ein hypothetisch-deduktives Vorgehen zur Konstruktion eines Fragebogens, der die Prozessmerkmale des gemäßigten Konstruktivismus nach Reinmann und Mandl (2006) erfasst. Sowohl das umfangreiche Messinventar von Urhahne et al. (2011) als auch die Kurzfassung von Basten et al. (2015) für einen ökonomischeren Einsatz im Unterricht erwiesen sich als messgenau. Daher kann die Kurzskala zur Messung gemäßigt konstruktivistischer Prozessmerkmale des zu untersuchenden Unterrichts eingesetzt werden. Wie bereits erörtert, sind hier- bei die Merkmale „aktiv“, „situativ“ und „konstruktiv“ von besonderem Interesse (s. „Konstruktivistische Prozessmerkmale fächerübergreifenden Unterrichts“). Zudem wurde eine sechsstufige Ratingskala zur Beantwortung der einzelnen Items gewählt. Die Subskala „konstruktiv“ wurde um das Item „... hat mir bisheriges Wissen weitergeholfen" ergänzt. - Tab. 1 zeigt die Subskalen und die internen Konsistenzen in Abhängigkeit der Unterrichtssettings.

Innerhalb der Subskalen konnten durchweg zufriedenstellende Reliabilitätswerte festgestellt werden, was für die Messgenauigkeit der sechsstufigen Ratingskala spricht (Döring \& Bortz, 2016).

Zur Beantwortung der vierten Hypothese wurde neben der Skala zur Erfassung der Prozessmerkmale des gemäßigten Konstruktivismus die Vorerfahrung auf einer vierstufigen Skala erfragt. Dazu sollten die Schüler ${ }^{\star}$ innen nach der fächerübergreifenden Unterrichtseinheit abschätzen, wie viel Vorwissen sie in Bezug zum Thema gehabt haben. Je zwei der Bewertungsstufen wurden in der neuen Variable „Vorwissen“ zu „wenig“ oder „viel“ Vorerfahrung zusammengefasst.

\section{Statistische Auswertung}

Das Projekt verfolgt das Design-based Research-Modell nach Shavelson, Phillips, Towne und Feuer (2003). Die Ergebnisse der vorgestellten Studie sind Resultat einer ersten Implementierung fächerübergreifender Unterrichtseinheiten, die in weiteren Schritten verfei- nert werden sollen. Zudem ermöglicht dieser Forschungsansatz die Fokussierung aus ersten Ergebnissen abgeleiteter Forschungsdesiderata. Die vorgestellten Subskalen sind Teil eines größeren Messinstruments, das für ein Prä-PostDesign genutzt wurde. So wurden die Schüler*innen vor und nach der fächerübergreifenden Unterrichtseinheit mit Hilfe des Messinstruments zu ihren Einstellungen befragt. Innerhalb dieser kleinen Teilstudie interessiert aber nur der zweite Testzeitpunt (Posttest), da dort die Prozessmerkmale des gemäßigten Konstruktivismus abgefragt wurden. Die Schüler*innen erhielten die Aussagen der einzelnen Subskalen und sollten sie auf einer sechsstufigen Skala im Hinblick auf die fächerübergreifende Unterrichtseinheit, ihren regulären Biologieunterricht und ihren regulären Sportunterricht bewerten. Die statistische Auswertung erfolgte über das Statistikprogramm IBM SPSS Statistics (IBM Corp., Armonk, NY, USA) in der Version 27.0. Zur Beantwortung der ersten Forschungsfrage wurde für jede Subskala eine einfaktorielle Varianzanalyse (ANOVA) mit Messwiederholung berechnet, um Unterschiede innerhalb der Unterrichtssettings zu untersuchen. Als Effektgrößenmaß wurde das partielle Eta-quadrat herangezogen. Aufgrund multipler Testung liegen die Ergebnisse Bonferroni korrigiert vor (Holm, 1979). Zur Beantwortung der zweiten Forschungsfrage wurde ein $t$-Test für unabhängige Stichproben gewählt, da ausschließlich der Einfluss des Vorwissens auf die Beurteilung der fächerübergreifenden Einheiten erfasst werden sollte. Da bei der Subskala „konstruktiv“ keine Homoskedastizität vorlag, wurde hierfür der Welch-Test genutzt. Cohens d diente in diesem Fall als Effektgrößenmaß. Auch für die $t$-Tests und den Welch-Test wurde die BonferroniHolm-Methode zur Adjustierung des $p$ Werts herangezogen (Holm, 1979). 


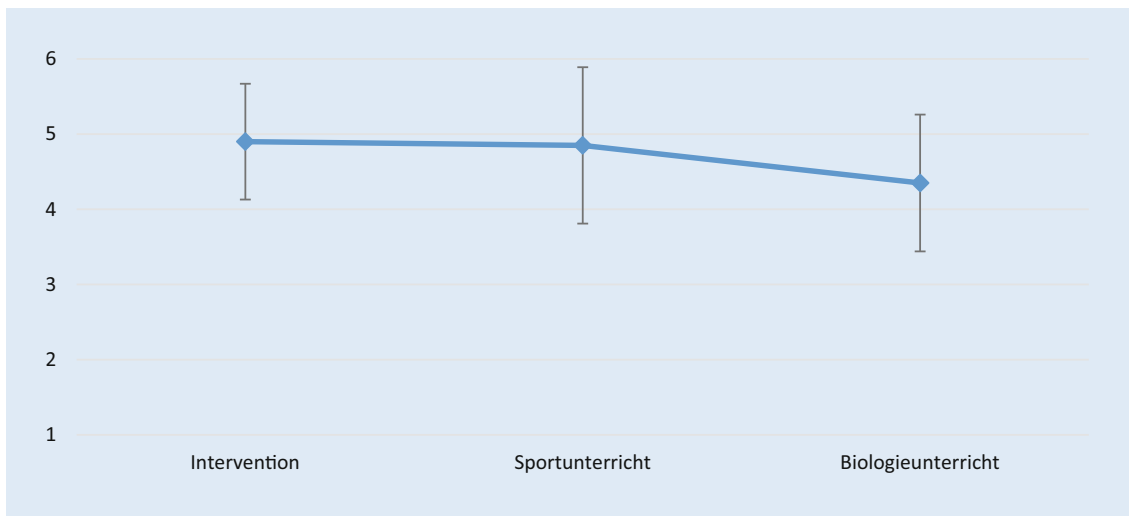

Abb. 2 \ Darstellung der Mittelwerte und Standardabweichungen des Konstrukts „aktiv“ in den dre Unterrichtssettings (fächerübergreifende Unterrichtseinheiten, Biologieunterricht, Sportunterricht; $\mathrm{n}=75$ )

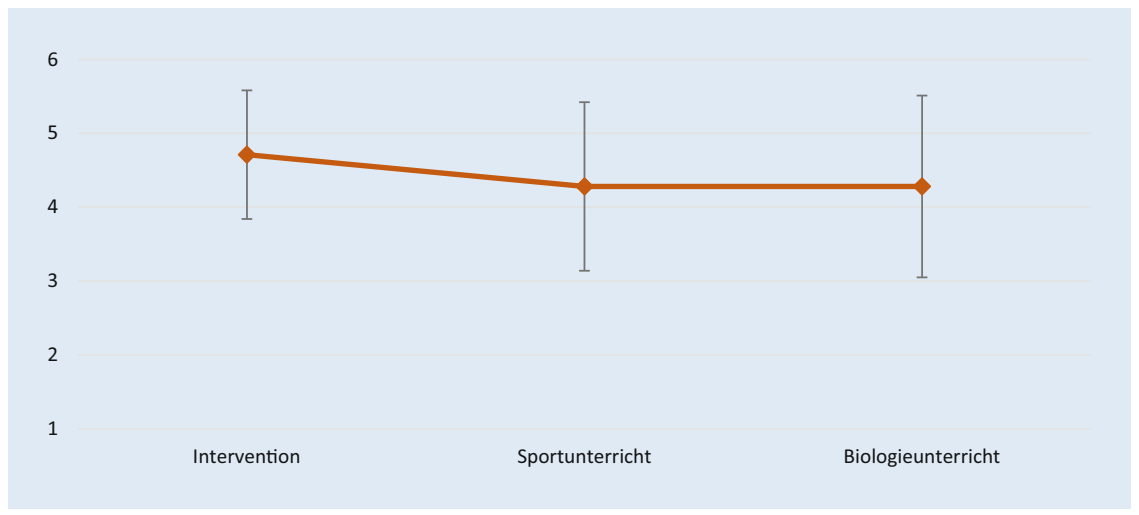

Abb. 3 A Darstellung der Mittelwerte und Standardabweichungen des Konstrukts "situativ“ in den drei Unterrichtssettings (fächerübergreifende Unterrichtseinheiten, Biologieunterricht, Sportunterricht; $n=75$ )

\section{Ergebnisse}

\section{Vergleich fächerübergreifender Unterrichtseinheiten mit dem Fachunterricht}

Zur Überprüfung der ersten Forschungsfrage und damit zur Prüfung der ersten Hypothesen (H1-H3) wurde für jede Subskala eine ANOVA mit Messwiederholung berechnet. Bei der Messwiederholung handelt es sich nicht um ein Prä-Post-Design, sondern um einen Vergleich der drei Treatments (Sportunterricht, Biologieunterricht, fächerübergreifender Unterricht). Die Ergebnisse werden nachfolgend mit Bezug zur deskriptiven Statistik dargestellt.

\section{Prozessmerkmal „aktiv“}

Innerhalb des Prozessmerkmals „aktiv“ $(n=75)$ konnte auf rein deskrip- tiver Ebene ein Unterschied zwischen den Unterrichtssettings festgestellt werden. Der Mittelwert liegt mit $M=4,90$ $(\mathrm{SD}=0,77)$ beim fächerübergreifenden Unterricht am höchsten. Danach folgt der Sportunterricht mit $M=4,85$ $(\mathrm{SD}=1,04)$ und der Biologieunterricht mit $\mathrm{M}=4,35$ ( $\mathrm{SD}=0,91$; 0 Abb. 2). Der Mauchly-Test ergab, dass eine Sphärizität angenommen werden kann (Mauchly$W(2)=0,927 ; p=0,063)$. Dabei zeigte sich ein signifikanter Unterschied zwischen den einzelnen Unterrichtssettings $(F(2,148)=11.170 ; \quad p \leq 0,001 ; \quad$ partielles $\left.\eta^{2}=0,131 ; n=75\right)$. Die Paarvergleiche belegten einen signifikanten Unterschied zwischen dem Biologieunterricht und den fächerübergreifenden Unterrichtseinheiten $(-0,55,95 \%$-KI $[-0,88$ bis $-0,22]$ ) sowie dem Sportunterricht $(-0,50,95 \%$-KI $[-0,83$ bis $-0,16])$. Im direkten Vergleich der fächerübergrei- fenden Unterrichtseinheiten und dem Sportunterricht konnte kein signifikanter Unterschied festgestellt werden $(0,05$; $95 \%$-KI [ $-0,22$ bis 0,32]). Demzufolge schätzen die Schüler*innen den Sportunterricht und den fächerübergreifenden Unterricht bezüglich des Prozessmerkmals „aktiv“ signifikant höher ein als ihren Biologieunterricht. Zwischen den fächerübergreifenden Unterrichtseinheiten und dem regulären Sportunterricht lässt sich kein Unterschied belegen.

\section{Prozessmerkmal „situativ“}

Für das Prozessmerkmal „situativ“ $(n=75)$ liegt der Mittelwert der fächerübergreifenden Unterrichtseinheiten $(\mathrm{M}=4,71, \mathrm{SD}=0,87)$ über denen des Sportunterrichts $(\mathrm{M}=4,28 ; \mathrm{SD}=1,14)$ und des Biologieunterrichts $(\mathrm{M}=4,28$; $\mathrm{SD}=1,23$; - Abb. 3). Bei näherer Betrachtung der Daten über die ANOVA (Sphärizität angenommen: Mauchly$W(2)=0,974 ; p=0,385)$ konnte ein signifikanter Unterschied zwischen den Unterrichtssettings festgestellt werden $(F(2,148)=5,169 ; \quad p=0,003 ;$ partielles $\left.\eta^{2}=0,077 ; n=75\right)$. Die Paarvergleiche ergaben einen signifikanten Unterschied zwischen dem fächerübergreifenden Unterricht und dem Biologieunterricht $(0,43 ; 95 \%-\mathrm{KI}[0,10-0,77])$ sowie dem Sportunterricht $(0,43 ; 95 \%-\mathrm{KI}$ $[0,10-0,77])$. Der Sportunterricht und der Biologieunterricht unterschieden sich hingegen nicht signifikant voneinander $(-0,002 ; 95 \%$-KI $[-0,38$ bis $0,37]$ ). Innerhalb des Prozessmerkmals „situativ" konnten konkludierend signifikant höhere Bewertungen der fächerübergreifenden Unterrichtseinheiten im Vergleich zu den Fächern Biologie und Sport festgestellt werden.

\section{Prozessmerkmal „konstruktiv“}

Beim Prozessmerkmal „konstruktiv“ $(n=75)$ wurde der höchste Mittelwert bei den fächerübergreifenden Unterrichtseinheiten ermittelt $(M=4,56 ; S D=0,99)$. Mit einem Unterschied von 0,09 Punkten folgt der Sportunterricht $(\mathrm{M}=4,45$; $\mathrm{SD}=0,90$ ), während der Biologieunterricht am niedrigsten bewertet wurde $(\mathrm{M}=4,27 ; \mathrm{SD}=1,10 ;-\mathrm{Abb} .4)$. Durch die ANOVA (Sphärizität angenommen: Mauchly- $W(2)=0,961 ; p=0,235)$ konn- 


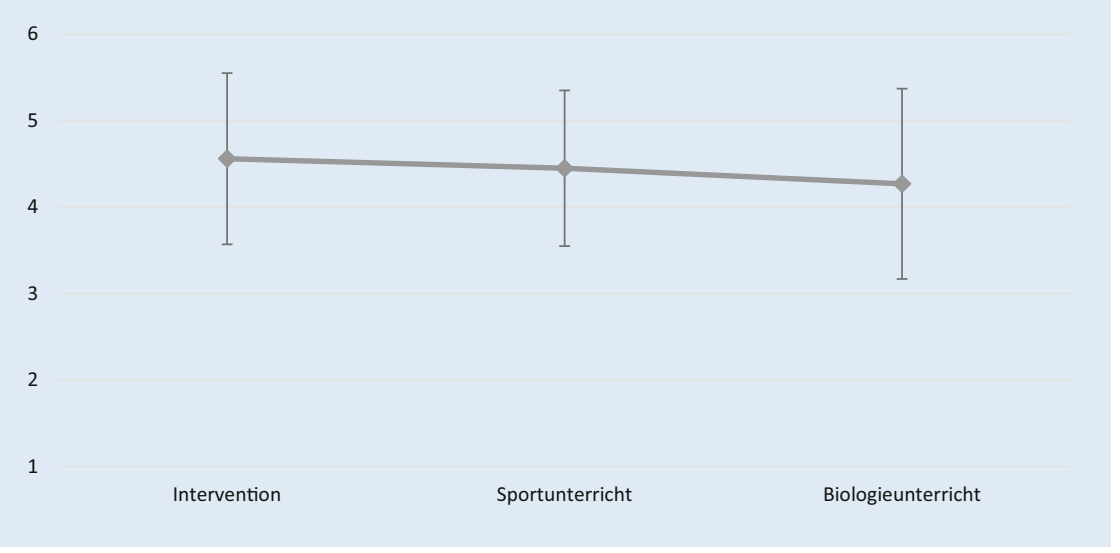

Abb. 4 A Darstellung der Mittelwerte und Standardabweichungen des Konstrukts "konstruktiv“ in den drei Unterrichtssettings (fächerübergreifende Unterrichtseinheiten, Biologieunterricht, Sportunterricht; $n=75$ )

te ein signifikanter Unterschied zwischen den einzelnen Unterrichtssettings gefunden werden $(F(2,148)=3,470 ; p=0,034$; partielles $\left.\eta^{2}=0,045 ; n=75\right)$. Die Ergebnisse der paarweisen Vergleiche legten einen Unterschied zwischen den fächerübergreifenden Unterrichtseinheiten und dem Biologieunterricht offen $(0,29 ; 95 \%$-KI $[-0,41$ bis 0,54$])$.

\section{Einfluss des Vorwissens}

Zur Beantwortung der zweiten Forschungsfrage wurde für jedes Konstrukt ein $t$-Test für unabhängige Stichproben herangezogen. Wie beschrieben, wurde für das Konstrukt „konstruktiv“ durch auftretende Heteroskedastizität ein Welch-Test berechnet. Insgesamt wurden $n=25$ Schüler*innen mit wenig Vorwissen $n=50$ Schüler ${ }^{\star}$ innen mit viel Vorwissen gegenübergestellt. Eine graphische Gegenüberstellung der einzelnen Mittelwerte findet sich in D Abb. 5 .

\section{Prozessmerkmal „aktiv“}

Für das Prozessmerkmal „aktiv“ lässt sich auf deskriptiver Ebene festhalten, dass jene Schüler*innen, die ein höheres Vorwissen in Bezug auf das Thema der Unterrichtseinheit angaben, ebenfalls das Konstrukt „aktiv“ höher bewerteten $(\mathrm{M}=4,93 ; \mathrm{SD}=0,68)$ als jene Schüler*innen, die wenig Vorwissen angaben $(\mathrm{M}=4,79 ; \mathrm{SD}=0,91)$. Der $t$-Test zeigt indes keinen signifikanten Unterschied zwischen den Gruppen $(t(73)=0,785$; $p=0,950)$.

\section{Prozessmerkmal "situativ"}

Ähnliche Ergebnisse konnten auch für das Konstrukt „situativ“ beschrieben werden. Der Mittelwert liegt innerhalb dieses Konstrukts bei der Gruppe mit viel Vorwissen $(\mathrm{M}=4,71 ; \mathrm{SD}=0,79)$ knapp vor dem der Gruppe, die weniger Vorwissen angab $(\mathrm{M}=4,69 ; \mathrm{SD}=0,99)$. Der Bonferroni-Holm korrigierte $t$-Test wies keinen signifikanten Unterschied aus $(t(73)=0,063 ; p=1)$.

\section{Prozessmerkmal „konstruktiv“}

Dasletzte Prozessmerkmal „konstruktiv“ bewerteten die Schüler*innen mit mehr Vorwissen höher $(\mathrm{M}=4,85 ; \mathrm{SD}=0,73)$ als jene Schüler ${ }^{\star}$ innen mit weniger Vorwissen $(M=3,94 ; S D=1,13)$. Beim WelchTest konnte ein signifikanter Unterschied zwischen den beiden Gruppen ermittelt werden $(t(34.283)=3,66 ; p=0,003)$. Die Bestimmung der Effektgröße über Cohens d ergab einen großen Effekt (Cohens $\mathrm{d}=$ 0,957; Cohen, 1988).

\section{Diskussion}

Reinmann und Mandl (2006) sprechen sich für die Umsetzung eines problemorientierten Unterrichts aus, um die sechs Prozessmerkmale gemäßigten Konstruktivismus in Schulen umsetzen zu können. Dieser Ansatz lässt sich im fächerübergreifenden Unterricht wiederfinden, da erst ein zu lösendes Problem das Zusammenwirken mehrerer Fachperspektiven initiiert (Kramer \& Wegner, 2020a). Daher ist die Problemorientierung fester
Bestandteil diverser Definitionen fächerübergreifenden Unterrichts (Moegling, 2010; Stübig, Bosse, \& Ludwig, 2008). Labudde (2003) argumentiert, dass vorrangig die Prozessmerkmale „aktiv“, „situativ" und „konstruktiv“ konstituierende Merkmale des fächerübergreifenden Unterrichts seien und sich dieser gerade in eben jenen Prozessmerkmalen vom Fachunterricht unterscheiden ließe. Für diese Annahme zeigen sich empirisch nur zum Teil stützende Befunde. Für das Konstrukt „situativ“ wird die fächerübergreifende Maßnahme signifikant höher eingeschätzt als der jeweilige Fachunterricht der Schüler*innen. Damit gelingt es innerhalb der Maßnahmen, von Schüler*innen als relevant eingestufte Themen zu behandeln, was im Fachunterricht in dieser Ausprägung nicht möglich ist. Die Hypothese H2 lässt sich demnach bestätigen. Dennoch sei angemerkt, dass die Schüler*innen ihren jeweiligen Fachunterricht ebenfalls überdurchschnittlich hoch einschätzen, weshalb es scheinbar im Sport- und Biologieunterricht ebenfalls gelingt, den Unterricht situativ $\mathrm{zu}$ gestalten. Für den Sportunterricht lässt sich dies damit begründen, dass thematisch häufig auf Situationen und Bewegungsmomente aus dem Alltag der Schüler ${ }^{\star}$ innen Bezug genommen wird, die den Unterricht situativ anbinden (vgl. „Konstruktivismus im Sport- und Biologieunterricht“). In der Biologie lässt sich dies beispielhaft durch die Behandlung humanbiologischer Themen erklären, die einen lebensrelevanten Kontext bieten. Doch auch dort finden sich abstraktere Themen, deren Kontext zwar hergestellt werden kann, aber nicht so intuitiv wie im Sportunterricht. Die Ergebnisse zeigen allerdings, dass sich beide Einzelfächer nicht voneinander unterscheiden. Bei Betrachtung der Subskala "aktiv“ lässt sich nur im Vergleich zum Biologieunterricht eine signifikant höhere Bewertung der fächerübergreifenden Maßnahmen feststellen. Dies bestätigt die Prämisse, dass im Sportunterricht eine aktive Auseinandersetzung mit dem Bewegungsgegenstand zwangsweise erforderlich ist und nicht von anderen erledigt werden kann, wodurch die hohe Bewertung dieses Prozessmerkmals begründet werden kann und die Sonder- 


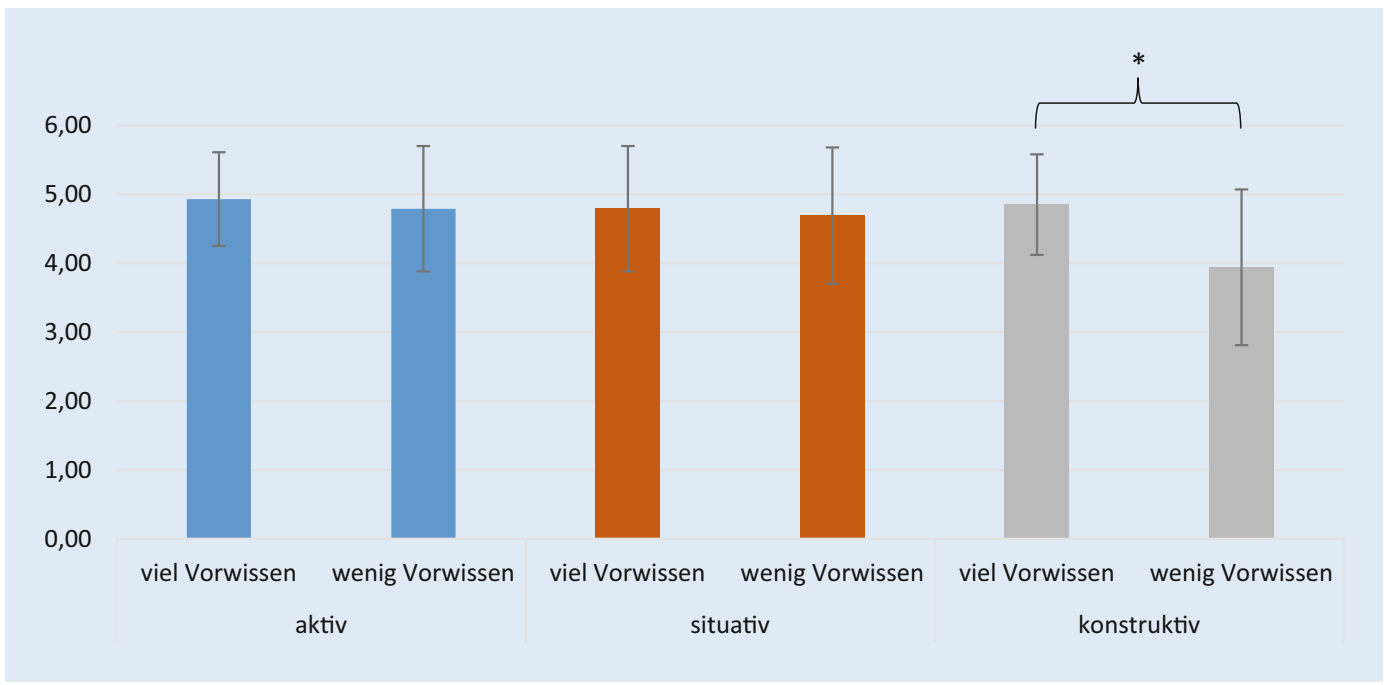

Abb. $5<$ Darstellung der Mittelwerte und Standardabweichungen bezüglich der Konstrukte „aktiv", "situativ" und "konstruktiv" unterschieden in Schüler*innen mit wenig $(n=25)$ und jenen mit viel Vorwissen $(n=50)$ stellung des Sportunterrichts unterstützt wird. Der Befund zeigt, dass dieses Prozessmerkmal nicht allein für den fächerübergreifenden Unterricht konstitutiv ist, sondern auch im regulären Fachunterricht Lernprozesse im gleichen Maße aktiv verlaufen können, ohne Fachgrenzen zu überschreiten. Gerade die aktive Auseinandersetzung mit Bewegungsproblemen im Sportunterricht weist in der Subskala starke Ähnlichkeiten zu den fächerübergreifenden Unterrichtseinheiten auf. Das Fach Biologie scheint in der fächerübergreifenden Maßnahme demnach der stärkste Profiteur zu sein, da die theoretischen Inhalte sportpraktisch und somit aktiv erlebbar werden, was im Fachraum in der Form nicht umsetzbar ist. Die Hypothese H1 ist daher nur für das Fach Biologie anzunehmen. Für das letzte Prozessmerkmal „konstruktiv“ wurde durch die ANOVA ein signifikanter Unterschied zwischen den fächerübergreifenden Unterrichtseinheiten und dem Biologieunterricht eruiert. Die deskriptive Statistik zeigt zudem einen Trend, der den Ergebnissen des Konstrukts „aktiv“ ähnelt. Vergleicht man die Strukturen, wird auch im Konstrukt „konstruktiv“ der Biologieunterricht am schlechtesten bewertet, wohingegen der Sportunterricht und die fächerübergreifenden Unterrichtseinheiten ähnliche Mittelwerte aufweisen. Ein Begründungsmoment lässt sich in der thematischen Breite der einzelnen Fächer finden. Der Sportunterricht wiederholt häufiger als der Biologieunter- richt ähnliche Themenbereiche und baut dadurch auf vorhandenes Wissen auf. Zudem machen Schüler*innen in ihrem Alltag zwangsweise neue motorische Erfahrungen, auf Grundlage derer sie im Sportunterricht neue Fertigkeiten und Fähigkeiten erlernen. Ein Unterschied zwischen dem fächerübergreifenden Unterricht und dem Fachunterricht findet sich aktuell lediglich für das Fach Biologie. Damit ist die Hypothese H3 nur für das Fach Biologie anzunehmen. Die ähnlich hohe Bewertung des Sportunterrichts im Vergleich zu den fächerübergreifenden Unterrichtseinheiten untermauert die Sonderstellung des Faches und zeigt, dass die Bewegungsprobleme scheinbar in konstruktiven Lernprozessen eingebunden werden. Die Ergebnisse suggerieren, dass die Biologie innerhalb der fächerübergreifenden Einheiten am meisten von dem Zusammenschluss mit dem Fach Sport profitiert, da sich dieses Fach am stärksten hinsichtlich der konstruktivistischen Merkmale unterscheidet. Besonders interessant erscheint dabei die Nähe des Sportunterrichts zum fächerübergreifenden Unterricht. Eine Erarbeitung von Bewegungsproblemen im Fachzusammenhang unterscheidet sich zumindest nicht subjektiv von einer fächerübergreifenden Auseinandersetzung mit einem Thema. Dennoch können sich beide konstruktivistischen Herangehensweisen in ihren Effekten unterscheiden. $\mathrm{Ob}$ durch eine fächerübergreifende Orientierung des Unterrichts die Motivation für das Einzelfach gesteigert oder ihm eine höhere Bedeutung beigemessen werden kann, muss in weiteren Studien überprüft werden. In diesem Zusammenhang und vor dem Hintergrund des im ersten Kapitel dargelegten Diskurses wäre ebenfalls interessant, inwiefern die Schüler*innen kognitiv aktiviert werden.

Neben der Charakterisierung der fächerübergreifenden Maßnahmen wurde geprüft, ob das Vorwissen einen Einfluss auf die Bewertung der konstruktivistischen Prozessmerkmale hat. Durch die Ergebnisse der $t$-Tests konnte dies nur für die Subskala „konstruktiv“ empirisch bestätigt werden. Die Schülerinnen mit subjektiv höherem Vorwissen bewerteten das Prozessmerkmal signifikant besser als die Schüler*innen mit geringerem Vorwissen. Dieser Unterschied ist nachvollziehbar, da durch die Subskala der Einbezug des Vorwissens in den Unterricht abgefragt wird. Ist nur sehr wenig Vorwissen vorhanden, kann es auch nicht miteinbezogen werden. Bei den anderen Subskalen wurde kein signifikanter Unterschied ermittelt. Das Vorwissen mag daher theoretisch einen Einfluss auf konstruktivistischen Unterricht haben. Die Ergebnisse dieser Studie zeigen aber, dass die Wahrnehmung der konstruktivistischen Prozessmerkmale durch die Schülerinnen nicht vom Vorwissen abhängen. Davon ausgenommen ist lediglich das Prozessmerkmal „konstruktiv“. Daher ist die Hypothese H4 bis auf die Subskala „konstruktiv“"vorerst zu verwerfen. Eine Limitation dieser Ergebnisse stellt 
die vierstufige Skala zur Bewertung des Vorwissens dar. Innerhalb der Stichprobe bewerteten die meisten Schüler ${ }^{\star}$ innen ihr Vorwissen im mittleren Bereich der Skala. Es gab also nur wenige Personen, die ihr Vorwissen am Rand der Skala einstuften. Um die Daten statistisch auszuwerten, wurden je zwei Stufen zusammengefasst, wodurch der Informationsgehalt teilweise verloren ging. Es ist wie auch schon bei der ersten Forschungsfrage zu empfehlen, eine größere Stichprobe zu untersuchen. Dadurch wäre es möglich, alle Bewertungsstufen der Skala statistisch einzubeziehen, wodurch der Einfluss des Vorwissens neu zu prüfen wäre.

\section{Ausblick}

Die vorliegende Studie konnte erste Unterschiede fächerübergreifenden Unterrichts im Fächerverbund Biologie und Sport im Vergleich zum jeweiligen Fachunterricht offenlegen. Es sind aber einige Limitationen wie die geringe Stichprobe anzuführen, weshalb eine weitere Untersuchung der Forschungsfragen $\mathrm{zu}$ empfehlen ist. Für eine zukünftige Auseinandersetzung wäre das Hinzuziehen einer Kontrollgruppe wichtig, um die Prozessmerkmale des gemäßigten Konstruktivismus im jeweiligen Fach oder zumindest losgelöst vom fächerübergreifenden Unterricht $\mathrm{zu}$ erfassen. Bisher konnte aber schon festgestellt werden, dass sich vor allen der Biologieunterricht von den fächerübergreifenden Unterrichtseinheiten unterschied, wohingegen die Prozessmerkmale des gemäßigten Konstruktivismus für den Sportunterricht ähnlich bewertet wurden. Dies unterstützt die Aussagen von Brodtmann und Landau (1982), die dem Fach eine Sonderstellung unter den Schulfächern hinsichtlich einer problemorientierten Ausrichtung und eine Nähe zum fächerübergreifenden Unterricht zusprechen. Das Vorwissen scheint bisher nur einen Einfluss auf das Prozessmerkmal „konstruktiv“ zu haben.

Eine weitere Überprüfung der postulierten Forschungsfragen ist durch die beschriebenen Limitationen dennoch wichtig und wünschenswert, um die aktuellen Befunde $\mathrm{zu}$ bestärken und weitere Effekte offenzulegen.

\section{Korrespondenzadresse}

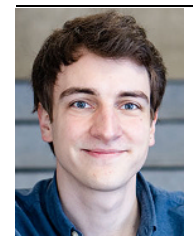

Niklas Kramer

Biologiedidaktik, Universität Bielefeld

Bielefeld, Deutschland

niklas.kramer@uni-

bielefeld.de

Förderung. Dieses Projekt wird im Rahmen der gemeinsamen „Qualitätsoffensive Lehrerbildung" von Bund und Ländern aus Mitteln des Bundesministeriums für Bildung und Forschung unter dem Förderkennzeichen 01JA1908 gefördert.

Funding. Open Access funding enabled and organized by Projekt DEAL.

\section{Einhaltung ethischer Richtlinien}

Interessenkonflikt. N. Kramer und C. Wegner geben an, dass kein Interessenkonflikt besteht.

Für diesen Beitrag wurden von den Autoren keine Studien an Menschen oder Tieren durchgeführt. Für die aufgeführten Studien gelten die jeweils dort angegebenen ethischen Richtlinien.

Open Access. Dieser Artikel wird unter der Creative Commons Namensnennung 4.0 International Lizenz veröffentlicht, welche die Nutzung, Vervielfältigung, Bearbeitung, Verbreitung und Wiedergabe in jeglichem Medium und Format erlaubt, sofern Sie den/die ursprünglichen Autor(en) und die Quelle ordnungsgemäß nennen, einen Link zur Creative Commons Lizenz beifügen und angeben, ob Änderungen vorgenommen wurden.

Die in diesem Artikel enthaltenen Bilder und sonstiges Drittmaterial unterliegen ebenfalls der genannten Creative Commons Lizenz, sofern sich aus der Abbildungslegende nichts anderes ergibt. Sofern das betreffende Material nicht unter der genannten Creative Commons Lizenz steht und die betreffende Handlung nicht nach gesetzlichen Vorschriften erlaubt ist, ist für die oben aufgeführten Weiterverwendungen des Materials die Einwilligung des jeweiligen Rechteinhabers einzuholen.

Weitere Details zur Lizenz entnehmen Sie bitte der Lizenzinformation auf http://creativecommons.org/ licenses/by/4.0/deed.de.

\section{Literatur}

Azzarito, L., \& Ennis, C. D. (2003). A sense of connection: toward social constructivist physical education. Sport, Education, and Society, 8, 179-197.

Balz, E. (2021). Perspektivierung und Aktivierung im Sportunterricht. In J. Wibowo, C. Krieger, E. Gerlach \& F. Bükers (Hrsg.), Aktivierung im Sportunterricht (2. Aufl. S. 14-22). Hamburg: Universität Hamburg.
Basten, M., Greiff, S., Marsch, S., Meyer, A., Urhahne, D., \& Wilde, M. (2015). Kurzskala zur Messung konstruktivistischer Prozessmerkmale im naturwissenschaftlichen Unterricht. Erkenntnisweg Biologiedidaktik, 14,43-57.

Brodtmann, D., \& Landau, G. (1982). An Problemen lernen.Sportpädagogik, 6(3), 16-22.

Chen, A., Martin, R., Sun, H., \& Ennis, C. D. (2007). Is physical activity at risk in constructivist physical education? Research Quarterly for Exercise and Sport, 78, 500-509.

Chen, S., Sun, H., Zhu, X., \& Chen, A. (2014). Relationship between motivation and learning in physical education and after-school physical activity. Research Quarterly for Sport and Exercise, 85(4), 468-477.

Cohen, J. (1988). Statistical power analysis for the behavioral sciences (2. Aufl.). Hillsdale: Lawrence Erlbaum.

Desch, I., Basten, M., Großmann, N., \& Wilde, M. (2017). Geschlechterdifferenzen in der wahrgenommenen Erfüllung der Prozessmerkmale gemäßigt konstruktivistischer Lernumgebungen - Die Effekte von Autonomieförderung durch Schülerwahl. Journal for Educational Research Online, 9(2), 156-182.

Döring, N., \& Bortz, J. (2016). Forschungsmethoden und Evaluation in den Sozial- und Humanwissenschaften (5. Aufl.). Springer-Lehrbuch. Berlin, Heidelberg:Springer.

Fernández-Balboa, J.-M. (1997). Physical education teacher preparation in the postmodern era: toward a critical pedagogy. In J.-M. Fernández-Balboa (Hrsg.), Critical postmodernism in human movement, physical education, and sport (S. 121-138). Albany: State University of New YorkPress.

Glaserfeld, E. v. (1984). An introduction to radical constructivism. In P. Watzlawick (Hrsg.), The invented reality (S. 17-40). New York: Norton.

Hermann, C., Seiler, S., \& Niederkofler, B. (2016). „Was ist guter Sportunterricht?" - Dimensionen der Unterrichtsqualität. sportunterricht, 65(3),7-12.

Holm, S. (1979). A simple sequentially rejective multiple test procedure. Scandinavian Journal of Statistics, 6(2), 65-70.

Hüfner, C., Mergelkuhl, T., Röllke, K., Kastrup, V., \& Wilde, M. (2016). Die Bewegung des Menschen: Fächerverbindend unterrichten. Unterricht Biologie, 40, 41-42.

KMK (Sekretariat der Ständigen Konferenz der Kultusminister der Länder in der Bundesrepublik Deutschland) (2021). Vereinbarung zur Gestaltung der gymnasialen Oberstufe und der Abiturprüfung. Bonn: KMK.

Kramer, N., \&Wegner,C. (2020a). Fächerübergreifender Unterricht im Fächerverbund Naturwissenschaften und Sport. Darstellung eines systematischen Reviews. Herausforderung Lehrer*innenbildung (HLZ), 3(1), 689-715.

Kramer, N., \& Wegner, C. (2020b). Herz-Kreislauf-System - Fächerübergreifende Aspekte entdecken und nutzen. sportunterricht, 69(1), 27-33.

Kramer, N., Großecosmann, A. M., \& Wegner, C. (im Druck). Lernen durch Bewegung - Gehirnjogging durch ein Training der exekutiven Funktionen. sportunterricht.

Krombholz, A., \& Fischer, B. (2018). Sachanalytische Betrachtung und Potenziale von Wassersport im schulischen Kontext. sportunterricht, 67(8), 339-343.

Labudde, P. (2003). Fächerübergreifender Unterricht in und mit Physik: eine zu wenig genutzte 
Chance. PhyDid A - Physik und Didaktik in Schule und Hochschule, 1(2), 48-66.

Labudde, P. (2008). Naturwissenschaften vernetzen, Horizonte erweitern: Fächerübergreifender Unterricht konkret. Seelze-Velber: Kallmeyer in Verbindung mit Klett.

Lipowsky, F. (2015). Unterricht. In E. Wild \& J. Möller (Hrsg.), Pädagogische Psychologie (S. 69-105). Berlin:Springer.

Lipowsky, F., \& Hess, M. (2019). Warum es manchmal hilfreich sein kann, das Lernen schwerer zu machen - Kognitive Aktivierung und die Kraft des Vergleichens. In K. Schöppe \& F. Schulz (Hrsg.), Kreativität \& Bildung - Nachhaltiges Lernen (S.77-132). München: kopaed.

Marsch, S., Hartwig, C., \& Krüger, D. (2009). Lehren und Lernen im Biologieunterricht - Ein Kategoriensystem zur Beurteilung konstruktivistisch orientierter Lernumgebungen. Zeitschrift Für Didaktik Der Naturwissenschaften, 15, 109-130.

Messmer, R. (2021). Lernen (im Sport) sichtbar machen. In J. Wibowo, C. Krieger, E. Gerlach \& F. Bükers (Hrsg.), Aktivierung im Sportunterricht (2. Aufl. S. 56-66). Hamburg: Universität Hamburg.

Ministerium für Schule und Weiterbildung des Landes Nordrhein-Westfalen (2014). Kernlehrplan für die Sekundarstufe II. Gymnasium/Gesamtschule in Nordrhein-Westfalen. Biologie. Düsseldorf: Ministerium für Schule und Weiterbildung des Landes Nordrhein-Westfalen.

Ministerium für Schule und Weiterbildung des Landes Nordrhein-Westfalen (2019). Kernlehrplan für die Sekundarstufe I. Gymnasium in NordrheinWestfalen. Biologie. Düsseldorf: Ministerium für Schule und Weiterbildung des Landes Nordrhein-Westfalen.

Moegling, K. (2010). Kompetenzaufbau im fächerübergreifenden Unterricht. Förderung vernetzten Denkens und komplexen Handelns. Immenhausen:Prolog.

Niederkofler, B., \& Amesberger, G. (2016). Kognitive Handlungsrepräsentationen als Strukturgrundlage zur Definition von kognitiver Aktivierung im Sportunterricht. Sportwissenschaft, 46(3), 188-200.

Popp, W. (1997). Die Spezialisierung auf Zusammenhänge als regulatives Prinzip der Didaktik. In L. Duncker \&W. Popp (Hrsg.), Grundlagen und Begründungen. Über Fachgrenzen hinaus: Chancen und Schwierigkeiten des fächerübergreifenden Lehrens und Lernens, (Bd. 1, S. 135-154). Heinsberg: Agentur Dieck.

Reich, K. (2005). Konstruktivistische Didaktik. Beispiele für eine veränderte Unterrichtspraxis. Schulmagazin 5-10, 3, 5-8.

Reinmann, G., \& Mandl, H. (2006). Unterrichten und Lernumgebungen gestalten. In A. Krapp \& B. Weidemann (Hrsg.), Pädagogische Psychologie (S.613-658). Weinheim: Beltz.

Shavelson, R. J., Phillips, D.C., Towne, L., \& Feuer, M. J. (2003). On the science of education design studies. Educational Researcher, 32(1), 25-28.

Stübig, F., Bosse, D., \& Ludwig, P.H. (2008). Problemorientierte Lehr-Lern-Arrangements in der Praxis - eine empirische Untersuchung zur Organisation und Gestaltung fächerübergreifenden Unterrichts. Zeitschrift für Pädagogik, 54(3), 376-395.

Sun, H., Chen, A., Zhu, X., \& Ennis, C.D. (2012). Learning science-based fitness knowledge in constructivist physical education. Elementary School Journal, 113(2), 215-229.

Sygusch, R., Hapke, J., Liebl, S., \& Töpfer, C. (2021). Kognitive Aktivierung - zentraler Baustein der
EKSpo-Aufgabenkultur zur Kompetenzorientierung im Sportunterricht. In J. Wibowo, C. Krieger, E. Gerlach \& F. Bükers (Hrsg.), Aktivierung im Sportunterricht (2. Aufl. S. 154-183). Hamburg: Universität Hamburg.

Terhart, E. (1999). Konstruktivismus und Unterricht. Gibt es einen neuen Ansatz in der Allgemeinen Didaktik? Zeitschrift für Pädagogik, 45(5), 629-647.

Ukley, N., Gröben, B., Wegner, C., \& Spintzyk, K. (2013). Fächerübergreifender Unterricht: Allgemeindidaktische Erwartungen. Fachdidaktische Anschlussmöglichkeiten. Beispielhafte Umsetzungsmöglichkeiten im Fach Sport. In A. Gogoll \& R. Messmer (Hrsg.), Sportpädagogik zwischen Stillstand und Beliebigkeit. 25. Jahrestagung der dvs-Sektion Sportpädagogik vom 7. bis 9. Juni 2012 (S. 139-152). Magglingen: Bundesamt für SportBASPO.

Urhahne, D., Marsch, S., Wilde, M., \& Krüger, D. (2011). Die Messung konstruktivistischer Unterrichtsmerkmale auf der Grundlage von Schülerurteilen. Psychologie in Erziehung und Unterricht, 58(2), 116-127.

Zhang, T., Chen, A., Chen, S., Hong, D., Loflin, J., \& Ennis, C.D. (2014). Constructing cardiovascular fitness knowledge in physical education. European Physical Education Review, 20(4), 425-443. 